\title{
Developments in the Application of Zernike Phase Contrast Technologies to Cryo- Electron Tomography Studies of Bacteria
}

\author{
E. R. Wright $^{1,2}$ and R. C. Guerrero-Ferreira ${ }^{1}$
}

${ }^{1}$ Emory University School of Medicine, Division of Pediatric Infectious Diseases, Children's Healthcare of Atlanta, 2015 Uppergate Drive NE, Atlanta, GA 30322

${ }^{2}$ Robert P. Apkarian Integrated Electron Microscopy Core, Emory University, 1521 Dickey Drive, Atlanta, GA 30322

Cryo-electron tomography (cryo-ET) is currently the only method available through which bacterial cells can be imaged to produce three-dimensional (3D) structures at $\sim 2$ to $10 \mathrm{~nm}$ resolution [1]. However, there still remain a few challenges associated with imaging whole bacteria by cryo-ET; namely the broad size range of bacteria; the low contrast of cryo-preserved, unstained specimens; and the requirement for low dose imaging to minimize specimen destruction. In an effort to overcome some of these limits, Zernike phase contrast (ZPC) transmission electron microscopy (TEM) was advanced [2]. ZPC cryo-TEM has been applied to studies of smaller macromolecular complexes and the thinnest edges of eukaryotic cells. Here we present results showing the success of using ZPC technologies for cryo-ET investigations of a substantially thicker specimen, specifically whole bacterial cells.

Caulobacter crescentus and Vibrio vulnificus strains were cultured overnight in appropriate media. Aliquots of $C$. crescentus and $V$. vulnificus cells were flash frozen onto glow-discharged Quantifoil carbon grids in liquid ethane with a FEI Mark III Vitrobot. Cryo-EM and cryo-ET data collection was performed with a JEOL JEM-2200FS $200 \mathrm{kV}$ FEG-TEM equipped with the Zernike phase plate airlock system located in the back-focal plane of the objective lens, an in-column energy filter (slit width 20 eV), a cryo-transfer specimen holder (Model 914, Gatan), and a 4k x 4k Gatan Ultrascan CCD camera. Images were acquired with a pixel size ranging from 0.55 to $1.1 \mathrm{~nm}$ on the specimen. For tilt series, a total electron dose between $30 \mathrm{e}^{-} / \AA^{2}$ and $120 \mathrm{e}^{-} / \AA^{2}$ was fractionated over tilt series ranging from $-62^{\circ}$ to $+62^{\circ}$. Tilt series images were taken automatically with $2^{\circ}$ tilt increments by using Serial EM [3].

In order to assess the application of ZPC for low dose image acquisition, we acquired duplicate images of multiple individual bacterial cells. One image was collected under defocus phase contrast (DPC) conditions and the other was acquired using ZPC. Each image was limited to an electron dose of $\sim 1.5$ or less $\mathrm{e}^{-} / \AA^{2}$, which corresponds to the dose of a single tilt series image. After examining the DPC and ZPC images of the bacterial cells (Fig. 1), we concluded that the ZPC images had improved SNR that allowed us to resolve features that were either barely discernable or undetectable in the equivalent DPC image. Some of the complexes unambiguously identified in the ZPC images include, the core filament of the flagellum, macromolecular complexes throughout the cytoplasm, and outer membrane vesicles and molecules contained within them. We further explored the potential of ZPC cryo-ET for the structural characterization of bacteria by analyzing multiple data sets of $C$. crescentus and $V$. vulnificus cells collected under both DPC and ZPC conditions at total electron doses of between $\sim 30 \mathrm{e}^{-} / \AA^{2}$ and $\sim 120 \mathrm{e}^{-}$ $/ \AA^{2}$. First, using ZPC settings, we reduced the total electron dose used to acquire a tilt series by $75 \%$ and generated high contrast, high SNR 3D reconstructions of thick bacterial cells. Second, we analyzed the relationship between total dose applied and the movement of the $10 \mathrm{~nm}$ gold fiducials used for image alignment. We determined that fiducial movement can be reduced by $\sim 50 \%$ when an electron dose of 
$\sim 30 \mathrm{e}^{-} / \AA^{2}$ is used for data collection, which will improve image alignment for the generation of higherquality tomographic reconstructions.

We have demonstrated that the thin carbon film ZPC technology allows one to reduce the electron dose applied to the specimen and maintain high contrast; achieve higher contrast in each tilt series image and in the final 3D reconstruction; improve image alignment due to the greater SNR of each image and the reduction of fiducial displacement; and reduce noise in the 3D volume. Future efforts in the field of improved phase contrast microscopy focus on the development of improved thin films, streamlining data collection schemes, and the implementation of airlock systems to upgrade existing instruments. Already, the use of thin carbon film ZPC significantly improves cryo-ET data of thicker specimens. Therefore, the continued development of the technology will increase the prospect of structural studies on a wider range of biological specimens.

\section{References:}

[1] J. L. Milne, S. Subramaniam. Nature Reviews. Microbiology 7(9) (2009), p. 666-675.

[2] R. Danev, K. Nagayama. Ultramicroscopy 88 (2001), p. 243-252.

[3] J. R. Kremer, D. N. Mastronarde, and J. R. McIntosh. 1996. Journal of Structural Biology 116(1) (1996), p. 71-76.

[4] This research was supported by funds from Emory University, Children's Healthcare of Atlanta, the Emory Center for AIDS Research, the Georgia Research Alliance, Human Frontiers Science Program, and the National Science Foundation to E.R.W. Cryo-EM/ET data was collected at the Emory University Robert P. Apkarian Integrated Electron Microscopy Core.
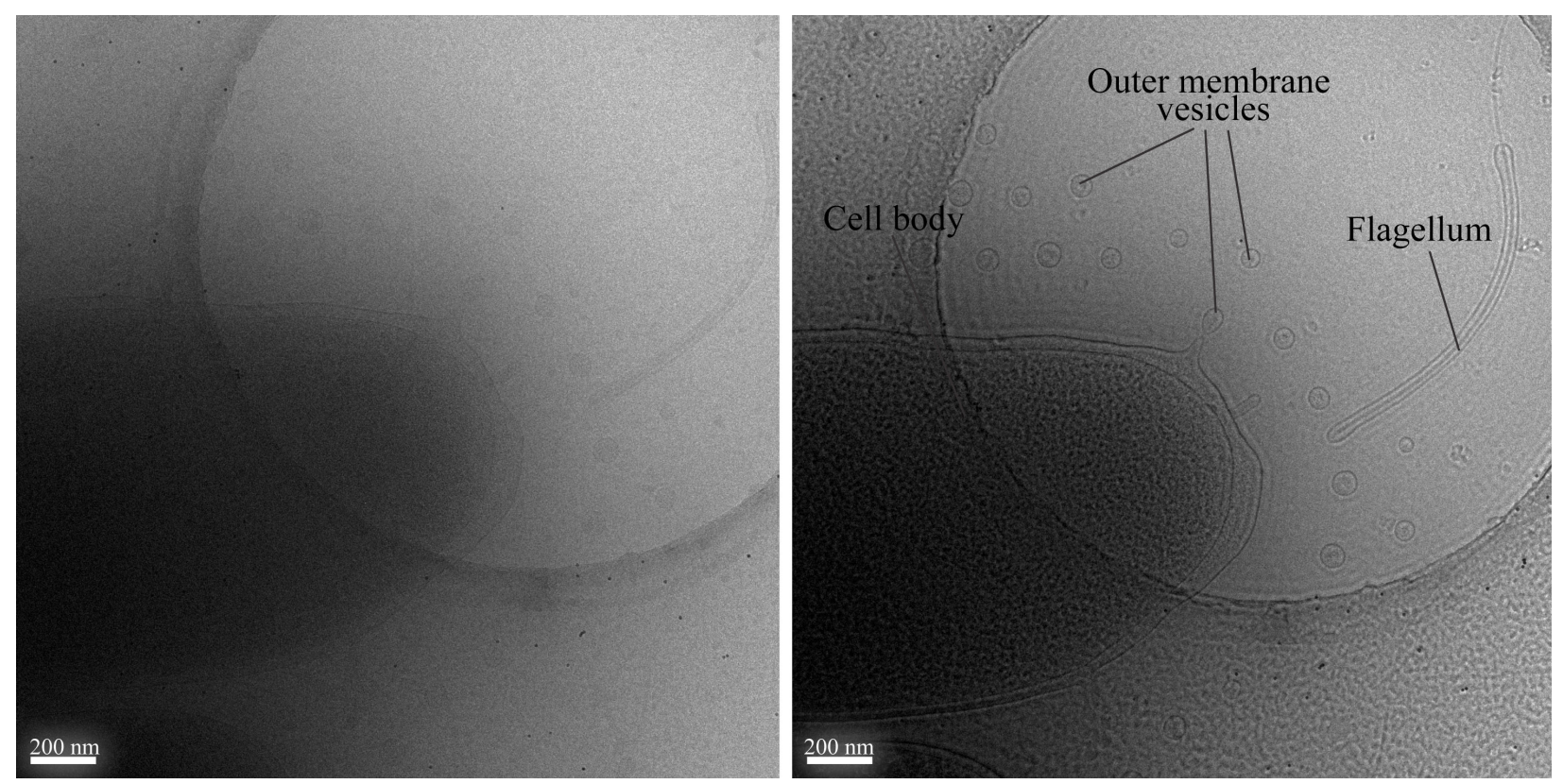

Figure 1. DPC and ZPC cryo-EM images of a frozen-hydrated bacterium. Single low dose $\left(\sim 1.5 \mathrm{e}^{-} / \AA^{2}\right)$ DPC (left) and ZPC (right) images of the same Vibrio vulnificus cell (cell body, flagellum, and outer membrane vesicles are identified). Scale bars, $200 \mathrm{~nm}$. 